\title{
Application of chemically amplified positive resist PSR for advanced mask making
}

\author{
Kazuo Kazama*, Fumio Murai**, Toshihiko Khono***, \\ Toshio Sakamizu**, and Shinji Okazaki** \\ * Hitachi Metal Ltd., Magnetic and Electronic \\ Materials Research Laboratory, \\ ** Hitachi, Ltd., Central Research Laboratory, \\ *** Hitachi, Ltd.,Total Production Operation
}

\section{Introduction}

Advanced technologies like phase-shift and modified illumination make it possible to delineate deep-submicron patterns by optical lithography. However these advanced optical technologies require precise mask fabrication technology. The development of electron beam resists is one of the key issues for advanced mask fabrication. Electron beam resists for the fabrication of precise masks must have both fine patterning capability and dry etching durability.

Chemically amplified positive resist PSR (Positive resist by Solubility Reversal) has been developed for direct wafer writing. 1) In this work, the application of PSR for mask writing is investigated.

\section{Experiment}

The e-beam resist used in this experiment is a three-component chemically amplified resist, consisting of an acid generator, novolak resin and tetrahydropyranyl-protected polyvinylphenol (THP-M) as a dissolution inhibitor. In the case of delineating insulating layers, such as a phase shifter layer, a water soluble conducting polymer ESPACER100 (Showa Denko Co.) was spun on top of the e-beam resist to prevent any charging problem.

The e-beam exposure system used in this work is HL750D (Hitachi Ltd.) with $50 \mathrm{kV}$ acceleration voltage. The current density and maximum shot size are $7 \mathrm{~A} / \mathrm{cm}^{2}$ and $2 \mu \mathrm{m}$ square, respectively.

\section{Results}

The sensitivity and resolution of chemically amplified resists are strongly dependent on the soft-bake and post-exposure-bake (PEB) conditions. In this experiment, two sets of baking conditions were adopted. To prevent an intermixing layer formation between the resist and the top-coating ESPACER100, a high soft-baking temperature of $125^{\circ} \mathrm{C}$ and a PEB temperature of $110^{\circ} \mathrm{C}$ were adopted. A low soft-baking temperature of $80^{\circ} \mathrm{C}$ and a PEB temperature of $80^{\circ} \mathrm{C}$ were adopted for delineation of the Chromium layer (TL-Cr). Figure 1 shows the sensitivity curves of these two baking conditions. 
J. Photopolvm. Sci. Technol., Vol.6, No.1, 1993

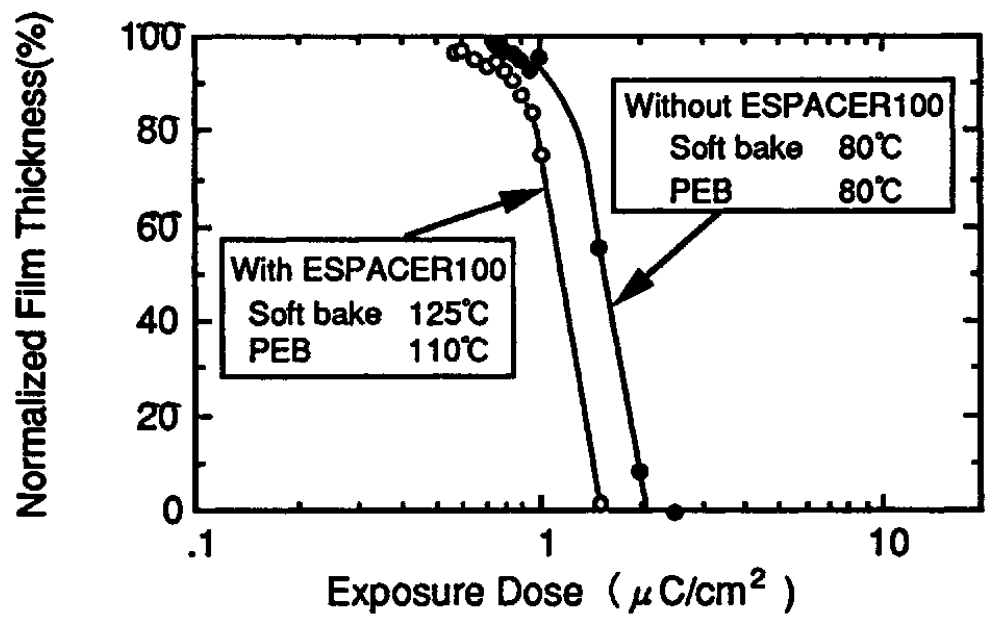

Fig.1 Sensitivity Characteristics of PSR

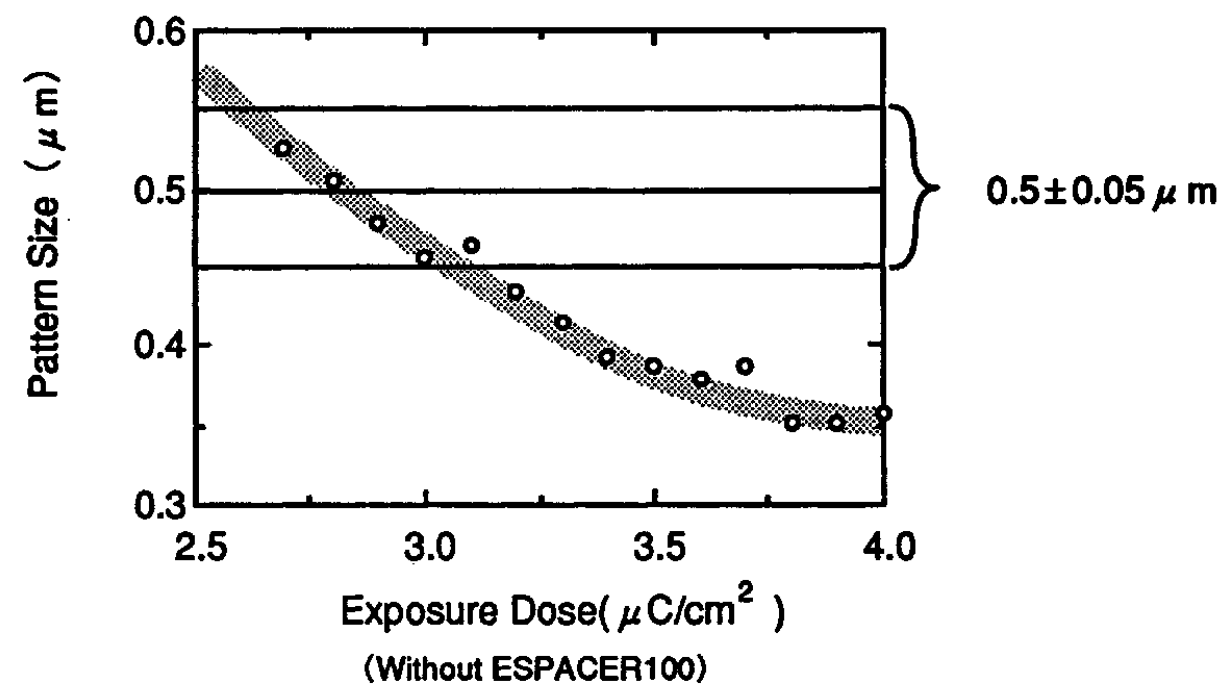

(a) Relationship between Line Pattern Size and Dose on $0.5 \mu \mathrm{m}$ Line/Space Patterns

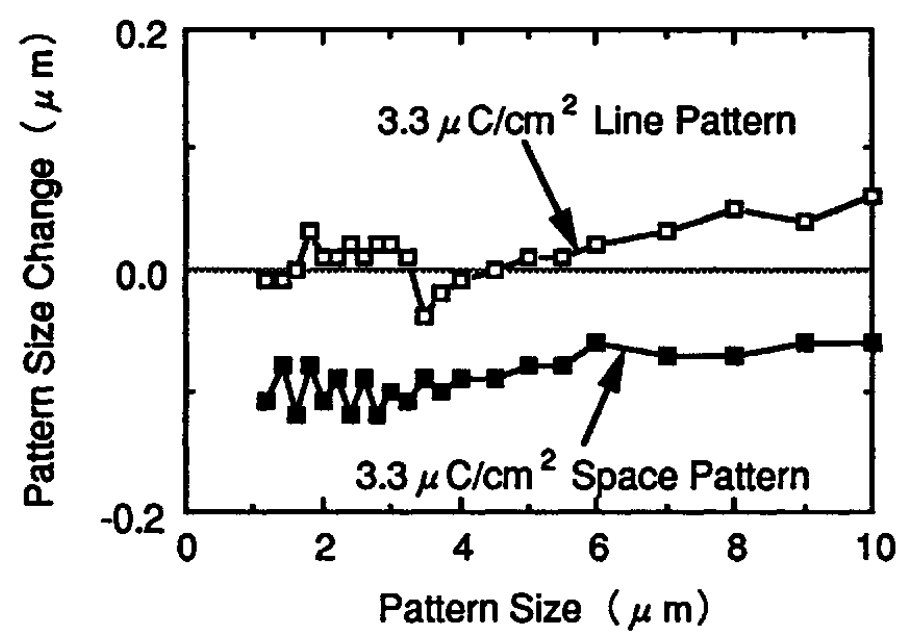

(b) Pattern Size Accuracy of Isolated Patterns

Fig.2 Pattern Size Accuracy 


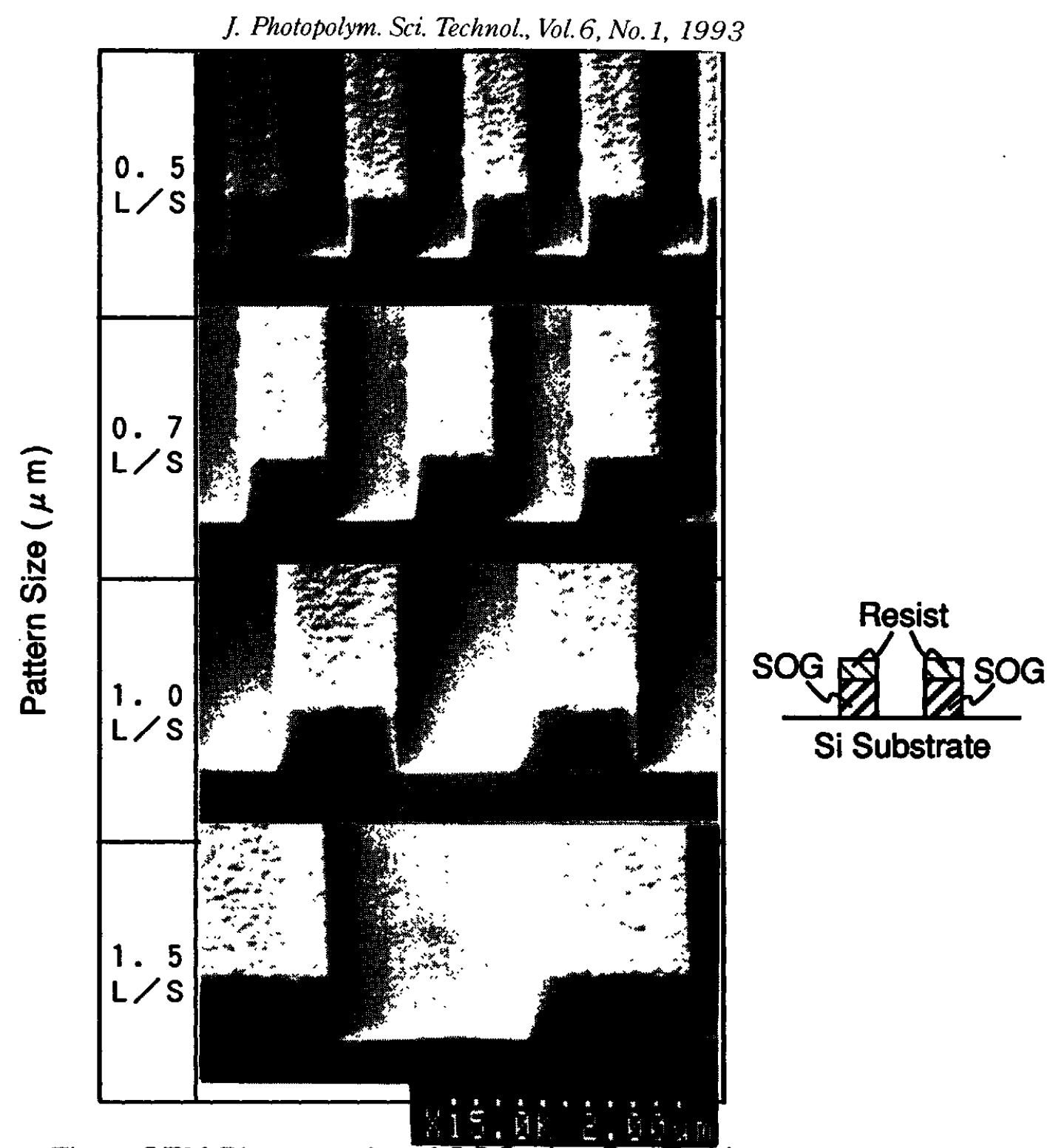

Fig.3 SEM Photographs of SOG after Dry Etching

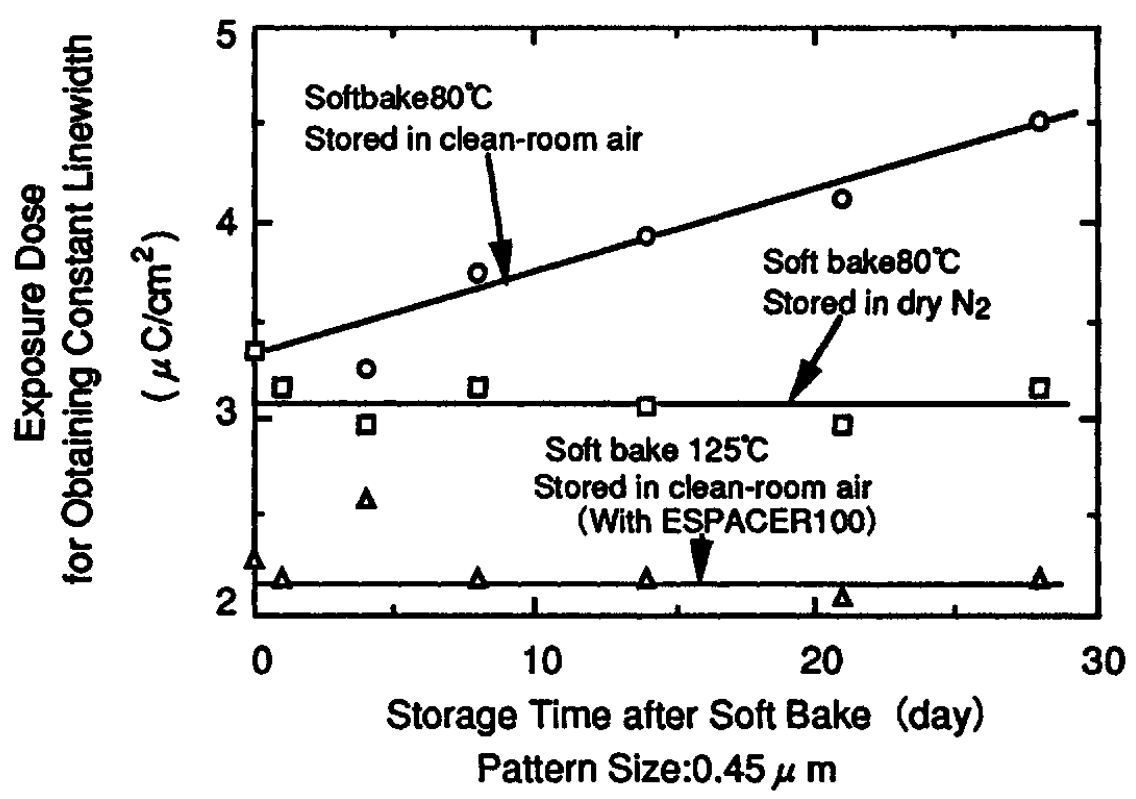

Fig.4 Stability of the Pre-Coated Film 
After etching the TL-Cr layer, pattern width was measured by LAMPAS IIi and pattern details were observed by SEM. Figure 2 shows the electron beam dose dependence of pattern size and pattern size change from the designed value. The sensitivity for a $0.5 \mu \mathrm{m}$ line/space pattern is around $3 \mu \mathrm{C} / \mathrm{cm}^{2}$ as shown in Fig. 2 . Pattern size accuracy can be controlled within $0.1 \mu \mathrm{m}$ in the range of 1.2 to $10 \mu \mathrm{m}$. As shown in Fig. 2(a) $10 \%$ change in the exposure dose causes only $0.026 \mu \mathrm{m}$ change in the $0.5 \mu \mathrm{m}$ line/space patterns.

In order to delineate the phase shifter layer, the dry etching durability of PSR was investigated. Film thickness of the shifter layer (SOG layer) was $0.4 \mu \mathrm{m}$, which corresponds to a $180^{\circ}$ phaseshift at the i-line. Figure 3 shows cross-sections of the dry-etched phase-shifter. This experiment used a microwave plasma etching system using a mixture gas of $\mathrm{C}_{4} \mathrm{~F}_{8} / \mathrm{CH}_{2} \mathrm{~F}_{2}$. The etching rate ratio of PSR to SOG was 1 to 2 under these conditions. Due to the strong etching resistance, very straight sidewalls can be obtained.

The delay time effect of chemically amplified resist is a serious problem. Especially for mask-making application, the stability of sensitivity in pre-coated resist film is important. Figure 4 shows the exposure dose change needed to maintain constant pattern size during store time. When a pre-coated resist film is stored in a clean-room atmosphere, some change in sensitivity is observed. However, when it is stored in dry nitrogen, the change can be negligible for at least 4 weeks of storage. It is also found that topcoated resist with ESPACER 100 is stable even in a clean-room environment.

\section{Conclusion}

The chemically amplified positive e-beam resist PSR was investigated for use in the mask-making process. Patterns of 0.5 $\mu \mathrm{m}$ critical dimension can be obtained with $3 \mu \mathrm{C} / \mathrm{cm}^{2}$ at $50 \mathrm{kV}$. Dry etching durability was also confirmed. To prevent sensitivity variation in PSR pre-coated film, a dry nitrogen atmosphere or over coat is necessary for long term storage. These results show that PSR is a promising positive e-beam resist for mask writing in the deepsubmicron range.

Acknowledgement

The authors wish to thank Dr. H. Shiraishi for resist preparation and helpful discussions. Thanks are also due to U. Soda for measuring pattern width.

Reference

1. T. Sakamizu, H. Shiraishi, H. Yamaguchi, T. Ueno, and N. Hayashi, Jpn. J. Appl. Phys. Vol.31 pp.4288-4293 Dec.1992 